\title{
Correction to: Human-as-a-security-sensor for harvesting threat intelligence
}

Manfred Vielberth", Florian Menges and Günther Pernul

\section{Correction to: Cybersecurity (2019) 2:23 \\ https://doi.org/10.1186/s42400-019-0040-0}

In the original publication of this article (Vielberth et al. 2019), the author list was not completed. The correct author list should be Manfred Vielberth, Florian Menges and Günther Pernul. The publisher apologizes to the readers and authors for the inconvenience.

The original publication has been corrected.

Published online: 26 November 2019

\section{Reference}

Vielberth et al (2019) Human-as-a-security-sensor for harvesting threat

intelligence. Cybersecurity 2:23. https://doi.org/10.1186/s42400-019-0040-0

\footnotetext{
* Correspondence: manfred.vielberth@ur.de

The original article can be found online at https://doi.org/10.1186/s42400019-0040-0

Universität Regensburg, Universitätstr. 31, 93053 Regensburg, DE, Germany
} 